\section{Morbid Obesity, Cardiac Disease, and Bariatric Surgery}

\author{
C. Richard Conti: Clin Cardiol 2007;589-590
}

\section{To the Editor:}

Dr. C. Richard Conti's insightful and authoritative December 2007 editorial $^{1}$ on bariatric surgery in patients with coronary diseases was read with much interest. At this point, I would request readers' attention to an uncommon emerging cardiac complication related to malabsorptive and restrictive surgeries undertaken in clinically severe obesity. There have been two recently reported cases of severe cardiomyopathy corroboratively related to low serum selenium levels that improved dramatically with selenium supplementation. The first case was reported (2006) from Australia in a 55-year-old female who underwent laparoscopic banding and then a laparoscopic biliopancreatic bypass 2 years later and subsequently presented after 9 months of the second procedure with chest tightness, severe breathlessness, gallop rhythm, effusions, nonspecific ST-T electrocardiogram (EKG) changes, no elevated cardiac enzymes, severe global systolic dysfunction (left ventricular ejection fraction $[\mathrm{LVEF}]=25 \%$ ), and no obstructive disease on coronary angiogram. ${ }^{2}$ Serum biochemistry showed widespread nutritional deficiencies (selenium, zinc, carotene, copper, vitamin A, vitamin E, selenium, and glutathione peroxidase), and her ejection fraction improved to $43 \%$ in 2 weeks with selenium replacement and angiotensinconverting enzyme (ACE) inhibition. The second reported patient, a 66-year-old female from Mayo Clinic (Rochester,
$\mathrm{MN}$ ), presented 3 years after a Roux-en-Y gastric bypass (a predominantly restrictive procedure) with acute left ventricular failure and cardiogenic shock. Her LVEF was 19\% with a concomitant serum selenium level of $41 \mathrm{ng} / \mathrm{mL}$ (normal, $95-165 \mathrm{ng} / \mathrm{mL}$ ), which improved to $62 \%$ in 2 monthd (repeat selenium level: $93 \mathrm{ng} / \mathrm{mL}$ ) after daily replacement $(50 \mathrm{mcg} / \mathrm{d})$ was initiated. ${ }^{3}$ Although there was evidence of other trace element deficiencies, cardiac decompensation was believed to be secondary to the selenium deficiency given its previously reported association and the temporal course. Close monitoring of nutritional deficiencies and replacement is indicated as a part of care after restrictive and malabsorptive procedures. ${ }^{4}$ Thus, as bariatric surgery may be a significant cause of the rarely reported nonendemic acquired selenium deficiency, ${ }^{2}$ awareness is warranted. Again, the risk of nutritional cardiomyopathy is minuscule (and reversible, too) compared with the immense cardiac (and noncardiac) advantages of surgery as mentioned in the editorial.

Nishith K. Singh, MD Department of Internal Medicine, Southern Illinois University School of Medicine, Springfield, Illinois

\section{References}

1. Conti CR: Morbid obesity, cardiac disease, and bariatric surgery. Clin Cardiol. 2007;30:589-590.

2. Boldery R, Fielding G, Rafter T, Pascoe AL, Scalia GM: Nutritional deficiency of selenium secondary to weight loss (bariatric) surgery associated with life-threatening cardiomyopathy. Heart Lung Circ. 2007; 16:123-126.

3. Harris S, Naina HV, Beckman TJ: Selenium deficiency and cardiomyopathy following bariatric surgery. Society of General Internal Medicine (SGIM) 30th Annual Meeting 2007.

4. DeMaria EJ: Bariatric surgery for morbid obesity. $N$ Engl J Med. 2007;356:2176-2183. 